\title{
ObliviSync: Practical Oblivious File Backup and Synchronization
}

\author{
Adam J. Aviv, Seung Geol Choi, Travis Mayberry, Daniel S. Roche \\ United States Naval Academy \\ \{aviv, choi, mayberry, roche\}@usna.edu
}

\begin{abstract}
Oblivious RAM (ORAM) protocols are powerful techniques that hide a client's data as well as access patterns from untrusted service providers. We present an oblivious cloud storage system, ObliviSync, that specifically targets one of the most widely-used personal cloud storage paradigms: synchronization and backup services, popular examples of which are Dropbox, iCloud Drive, and Google Drive. This setting provides a unique opportunity because the above privacy properties can be achieved with a simpler form of ORAM called write-only ORAM, which allows for dramatically increased efficiency compared to related work. Our solution is asymptotically optimal and practically efficient, with a small constant overhead of approximately $4 x$ compared with non-private file storage, depending only on the total data size and parameters chosen according to the usage rate, and not on the number or size of individual files. Our construction also offers protection against timing-channel attacks, which has not been previously considered in ORAM protocols. We built and evaluated a full implementation of ObliviSync that supports multiple simultaneous read-only clients and a single concurrent read/write client whose edits automatically and seamlessly propagate to the readers. We show that our system functions under high work loads, with realistic file size distributions, and with small additional latency (as compared to a baseline encrypted file system) when paired with Dropbox as the synchronization service.
\end{abstract}

\section{INTRODUCTION}

ORAM: security and efficiency. ORAM is a protocol which allows a client to access files (commonly abstracted as $N$ fixed-length blocks of data) stored on an untrusted server in such a way that the server learns neither the contents of files nor the access patterns of which files were accessed at which time(s). This is traditionally accomplished by doing some type of shuffling on the data in addition to reading/writing the chosen block. This shuffling ensures that the server cannot correlate logical blocks based on their storage locations.

ORAM is a powerful tool that solves a critical problem in cloud security. Consider a hospital which uses cloud storage to backup their patient records. Even if the records are properly encrypted, an untrusted server that observes which patient files are modified will learn sensitive medical information about those patients. They will certainly learn that the patient has visited the hospital recently, but also may learn things like whether the patient had imaging tests done based on how large the file is that is updated. Moreover, they might learn for instance that a patient has cancer after seeing an oncologist update their records. This type of inference, and more, can be done despite the fact that the records themselves are encrypted because the access pattern to the storage is not hidden.

Unfortunately, in order to achieve this obliviousness
ORAMs often require a substantial amount of shuffling during every access, so much so that even relatively recent ORAM constructions could induce a several-thousand-fold overhead on communication [22], [18]. Even Path ORAM [23], one of the most efficient ORAM constructions to date, has a practical overhead of 60-80x on moderately sized databases compared to non-private storage.

The setting: personal cloud storage. Our setting consists of an untrusted cloud provider and one or more clients which backup data to the cloud provider. If there are multiple clients, the cloud provider propagates changes made by one client to all other clients, so that they each have the same version of the filesystem. We emphasize that although we may use "Dropbox" as a shorthand for the scenario we are addressing, our solution is not specific to Dropbox and will work with any similar system. This setting is particularly interesting for a number of reasons:

1) It is one of the most popular consumer cloud services used today, and is often colloquially synonymous with the term "cloud." Dropbox alone has over 500 million users [12].

2) The interface for Dropbox and similar storage providers is "agnostic," in that it will allow you to store any data as long as you put it in the designated synchronization directory. This allows for one solution that works seamlessly with all providers.

3) Synchronization and backup services do not require that the ORAM hide a user's read accesses, only the writes. This is because (by default) every client stores a complete local copy of their data, which is synchronized and backed up via communication of changes to/from the cloud provider.

Our goal. In this paper, we present an efficient solution for oblivious storage on a personal cloud synchronization/backup provider such as (but not limited to) Dropbox or Google Drive.

Write-only ORAM. The third aspect of our setting above (i.e., we don't need to hide read accesses) is crucial to the efficiency of our system. Each client already has a copy of the database, so when they read from it they do not need to interact with the cloud provider at all. If a client writes to the database, the changes are automatically propagated to the other clients with no requests necessary on their part. Therefore, the ORAM protocol only needs to hide the write accesses done by the clients and not the reads. This is important because [8] have shown that write-only ORAM can be achieved with optimal asymptotic communication overhead of $O(1)$. In practice, write-only ORAM requires only a small constant overhead of 
3-6x compared to much higher overheads for fully-functional ORAM schemes, which asymptotically are $\Omega(\log N)$.

Note that Dropbox (and other cloud providers) do have client software that allows retrieval of only individual files or directories, for instance the Google Drive web interface. However, to achieve privacy in those settings with partial synchronization would require the full functionality of Oblivious RAM that hides both reads and writes. We instead specifically target the full-synchronization setting for two reasons:

1) It is the default behavior for the desktop clients of Dropbox, Google Drive, OneDrive, and others, making it a widely used, practical scenario.

2) Read-write ORAMs are subject to a well-known lower bound of $\Omega(N \log N)$ [15]. We aim to show that in a synchronization setting substantially better performance can be achieved that rivals the performance of insecure storage.

Providing cloud-layer transparency. One of the most noteworthy aspects of Dropbox-like services is their ease of use. Any user can download and install the requisite software, at which point they have a folder on their system that "magically" synchronizes between all their machines, with no additional setup or interaction from the user. In order to preserve this feature as much as possible, our system implements a FUSE filesystem that mounts on top of the shared directory, providing a new virtual frontend directory where the user can put their files to have them privately stored on the cloud. The FUSE module uses the original shared directory as a backend by storing blocks as individual files. We stress that substantial work is needed to add support for filesystem features such as filenames and sizes, since the storage of ORAMs is traditionally modeled as only a flat array of $N$ fixed-length blocks indexed by the numbers $[0, N)$.

Supporting variable-size files. When addressing the personal cloud setting, a crucial aspect that must be dealt with is the variable sizes of the files stored in such a system. Traditionally, ORAMs are modeled as storage devices on $N$ fixed-length blocks of data, with the security guarantee being that any two access patterns of the same length are indistinguishable from each other. In reality, files stored on Dropbox are of varying (sometimes unique) lengths. This means that a boilerplate ORAM protocol will actually not provide obliviousness in such a setting because the file size, in multiples of the block size, will be leaked to the server for every access. When file sizes are relatively unique, knowing the size will enable the server to deduce which individual file is being accessed, or at least substantially reduce the number of possibilities. Therefore our solution additionally includes a mechanism for dynamically batching together variable-length files to hide their size from the server. Furthermore, our solution is efficient as we prove its cost scales linearly with the total size (and not number) of files being written, regardless of the file size distribution. The batching aspect of our construction also allows us to protect against timing-channel attacks (where the precise time of an access leaks information about it), which are not usually considered in ORAM protocols.

Summary of our contribution. To summarize, our contributions in this paper include:
1) A complete ORAM system designed for maximum efficiency and usability when deployed on a synchronization/backup service like Dropbox.

2) A FUSE implementation of these contributions, incorporating variable size files as well as important filesystem functionality into ORAM including the ability to store file names, resize files and more.

3) A proof of strong security from an untrusted cloud provider, even considering the timing side-channel.

4) Theoretical evaluation showing that the throughput of our scheme requires only $4 \mathrm{x}$ bandwidth overhead compared to that of unencrypted and non-private storage, regardless of the underlying file size distribution ${ }^{1}$. We also show that our scheme has very high storage utilization, requiring only $1.5-2.0 \mathrm{x}$ storage cost overhead in practice.

5) An empirical evaluation of the system that shows that ObliviSync performs better than the theoretic results for both throughput and latency, and ObliviSync functions with limited overheads and delays when working with Dropbox as the cloud synchronization service.

\section{EFFICIENT ObLIVIOUSNESS FOR DROPBOX}

\section{A. Overview of Write-only ORAM}

We start by describing the write-only ORAM of [8], as it informs our construction.

The setting. To store $N$ blocks in a write-only ORAM, the server holds an array of $2 N$ encrypted blocks. Initially, the $N$ blocks of data are shuffled and stored in random locations in the $2 N$-length array, such that half of the blocks in the array are "empty". However, every block is encrypted with an IND-CPA encryption scheme so the server cannot learn which blocks are empty and which are not. The client stores a local dictionary (or sometimes called a position map) which maps a logical address in the range $(0, N]$ to the location in the server array where it is currently stored, in the range $(0,2 N]$. Using this dictionary, the client can find and read any block in the storage that it needs, but the server will not know the location of any individual block.

Read and write operations. Since by definition a write-only ORAM does not need to hide reads, they are performed trivially by reading the local dictionary and then the corresponding block from the ORAM. Write operations, however, require additional work. When the client wishes to write a block to the ORAM, it chooses $k$ random locations in the array out of $2 N$, where $k$ is a constant parameter. With high probability, at least one out of these $k$ locations will be empty, and the new block is written into that location while re-encrypting the other $k-1$ locations to hide which block was changed. After writing the block, the client also updates their dictionary to indicate that the block now resides in its new location. The old location for this block is implicitly marked empty because no entry in the dictionary now points to it.

Achieving obliviousness. Since every write operation sees the client accessing $k$ randomly chosen blocks in the ORAM,

\footnotetext{
${ }^{1}$ The bandwidth is actually set directly according to the system parameters. If it is too high, "dummy" write operations are performed to hide the access pattern. Our system works as long as the bandwidth is set to $4 \mathrm{x}$ higher than the actual amount of data written. Of course, the user may set the parameters poorly due to not knowing their usage in advance, in which case the bandwidth may be higher due to the required dummy writes. See Section II-B.
} 
independent of the logical address of the block that is being written, it cannot reveal any information about the client's access pattern. The only situation that can cause the client to reveal something is if the $k$ chosen locations do not contain any free blocks, and it has nowhere to write the new one. Since every block has $1 / 2$ probability of being empty, the chance that there are no free blocks will be $2^{-k}$, so $k$ can be set to the security parameter $\lambda$ to give a negligible chance of failure.

Efficiency with stash on the client. However, setting $k=\lambda$ actually does not result in $O(1)$ overhead; since $\lambda>\log N$, the overhead is $\Omega(\log N)$. On average, the client finds $k / 2$ empty blocks during a single write, many more than are needed. If the client instead stores a buffer of blocks that it wants to write, and writes as many blocks from the buffer as he finds empty blocks, $k$ can be set much more aggressively. It is shown in [8] that $k=3$ is sufficient to guarantee with high probability that the stash will never exceed $O(\log N)$. This makes the final overhead for write-only ORAM 3x that of non-private storage.

Maintaining the dictionary file. The final important detail is that the dictionary file requires $O(N \log N)$ bits of storage, which might be too large for the client to store locally. Fortunately it is relatively simple to store this dictionary recursively in another ORAM [8], [23]. For some block and databases sizes, however, it might be quite reasonable for the client to store the entire dictionary itself. Jumping ahead, in our system, the client locally stores the dictionary file (called the filetable) as an important metadata structure of the entire file system, in order to keep track of the actual position of each file block. See the detailed discussion in Section IV-A.

\section{B. Overview of Our System}

The setting. Our ObliviSync system uses the idea of writeonly ORAM on top of any file backup or synchronization tool in order to give multiple clients simultaneous updated access to the same virtual filesystem, without revealing anything at all to the cloud service that is performing the synchronization itself, even if the cloud service is corrupted to become an honest-but-curious adverary. Write-only ORAM is ideal for this setting because each client stores an entire copy of the data, so that only the changes (write operations) are revealed to the synchronization service and thus only the write operations need to be performed obliviously.

Improvements over write-only ORAM. Compared to the previous write-only ORAM construction [8], we make significant advances and improvements to fit this emergent application space:

- Usability: Users interact with the system as though it is a normal system folder. All the encryption and synchronization happens automatically and unobtrusively.

- Flexibility: We support a real filesystem and use innovative methods to handle variable-sized files and changing client roles (read/write vs. read-only) to support multiple users.

- Strong obliviousness: The design of our system not only provides obliviousness in the traditional sense, but also protects against timing channel attacks. It also conceals the total number of write operations, a stronger guarantee than previous ORAM protocols.

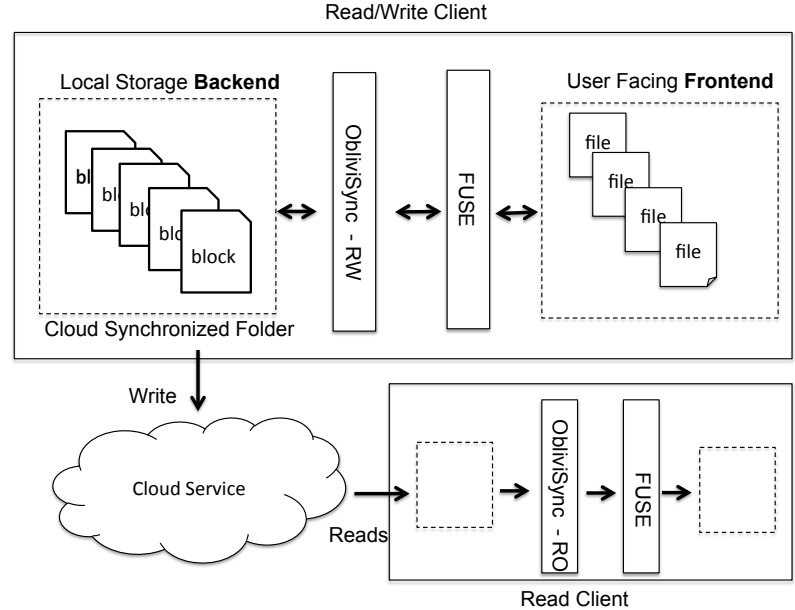

Fig. 1. Diagram for ObliviSync

- Performance: Our system well matches the needs of real file systems and matches the services provided by current cloud synchronization providers. It can also be tuned to different settings based on the desired communication rate and delay in synchronization.

Basic architecture. The high-level design of ObliviSync is presented in Figure 1. There are two types of clients in our system: a read/write client (ObliviSync-RW) and a read-only client (ObliviSync-RO). At any given time, there can be any number of ObliviSync-RO's active as well as zero or one ObliviSync-RW clients. We note that a given device may work as a read-only client in one period of time and as a writeonly client in other periods of time. ${ }^{2}$ Both clients consist of an actual backend folder as well as a virtual frontend folder, with a FUSE client running in the background to seamlessly translate the encrypted data in the backend to the user's view in the frontend virtual filesystem.

We rely on existing cloud synchronization tools to keep all clients' backend directories fully synchronized. This directory consists of encrypted files that are treated as generic storage blocks, and embedded within these storage blocks is a file system structure loosely based on i-node style file systems which allows for variable-sized files to be split and packed into fixedsize units. Using a shared private key (which could be derived from a password) the job of both clients ObliviSync-RO and ObliviSync-RW is to decrypt and efficiently fetch data from these encrypted files in order to serve ordinary read operations from the client operating in the frontend directory.

The ObliviSync-RW client, which will be the only client able to change the backend files, has additional responsibilities: (1) to maintain the file system encoding embedded within the blocks, and (2) to perform updates to the blocks in an oblivious manner using our efficient modification of the writeonly ORAM described in the previous subsection.

User transparency with FUSE mount. From the user's perspective, however, the interaction with the frontend directory

\footnotetext{
${ }^{2}$ How to make sure that only one write-only client operates at a given time is out the scope, and in this paper, we will simply assume the existence of the procedure to enforce it.
} 
occurs as if interacting with any files on the host system. This is possible because we also implemented a FUSE mount (file system in user space) interface which displays the embedded file system within the backend blocks to the user as if it were any other file system mount. Under the covers, though, the ObliviSync-RO or ObliviSync-RW clients are using the backend directory files in order to serve all data requests by the client, and the ObliviSync-RW client is additionally monitoring for file changes/creations in the FUSE mount and propagating those changes to the backend.

Strong obliviousness through buffered writes. In order to maintain obliviousness, these updates are not immediately written to the backend filesystem by the ObliviSync-RW client. Instead, the process maintains a buffer of writes that are staged to be committed. At regular timed intervals, random blocks from the backend are loaded, repacked with as much data from the buffer as possible, and then re-encrypted and written back to the backend folder. From there, the user's chosen file synchronization or backup service will do its work to propagate the changes to any read-only clients. Moreover, even when there are no updates in the buffer, the client pushes dummy updates by rewriting the chosen blocks with random data. In this way, as the number of blocks written at each step is fixed, and these writes (either real or dummy) occur at regular timed intervals, an adversary operating at the network layer is unable to determine anything about the file contents or access patterns. Without dummy updates, for example, the adversary can make a reasonable guess about the size of the files that the client writes; continuted updates without pause is likely to indicate that the client is writing a large file. Note that in some cases, revealing whether a client stores large files (e.g., movies) may be sensitive. Further details on all of these components can be found in Section IV. The full source code of our implementation is available on GitHub [14].

\section{SECURITY DEFINITIONS}

\section{A. Write-only Oblivious Synchronization}

Block-based filesystem. Our system has more capabilities than a standard ORAM, including support for additional filesystem operations, so we require a modified security definition which we present here. We first formally define the syntax of a block-based filesystem with block size $B$.

- create(filename): create a new (empty) file.

- delete(filename): remove a file from the system.

- resize(filename,size): change the size of a file. The size is given in bytes, and can be greater or smaller than the current size.

- write(filename,offset,length): write data to the identified file according to the offset and length arguments. The offset is a block offset. Unless the offset refers to the last block in the file, length must be a multiple of $B$.

- read(filename,offset,length) $\rightarrow$ data: read data from the identified file according to the offset and length arguments. Again, offset is a block offset, and length must be a multiple of the block size $B$ unless the read includes the last offset.

For simplicity, we only consider these five core operations. Other standard filesystem operations can be implemented using these core functionalities.
Obliviousness and more. The original write-only ORAM definition in [8] requires indistinguishability between any two write accesses with same data sizes. However, the definition does not consider the time at which write operations take place. Here, we put forward a stronger security notion for the file system that additionally hides both the data length and the time of non-read operations.

For example, we want to make sure all the following operation sequences are indistinguishable:

- no write operations at all

- write(file1,1,5) and write(file2,3,3) at time 3, and write(file $1,6,9)$ at time 5

- write(file $2,1,20)$ at time 5

For this purpose, we first define $(L, t)$-fsequences. Here, the parameter $L$ is the maximum number of bytes that may be modified, and $t$ is the latest time that is allowed. For example, the above sequences are all $(20,5)$-fsequences, since all sequences write at most 20 bytes data in total and have the last write before or at time 5 .

Definition $1((L, t)$-fsequence). A sequence of non-read operations for a block filesystem is a $(L, t)$-fsequence if the total number of bytes to be modified in the filesystem metadata and file data is at most L, and the last operation takes place before or at time $t$.

Our goal is to achieve an efficient block filesystem construction such that any two $(L, t)$-fsequences are indistinguishable.

Definition 2 (Write-only strong obliviousness). Let $L$ and $t$ be the parameters for fsequences. A block filesystem is writeonly strongly-oblivious with running time $T$, if for any two $(L, t)$-fsequences $P_{0}$ and $P_{1}$, it holds that:

- The filesystem finishes all the tasks in each fsequence within time $T$ with probability $1-n e g(\lambda)$, where is $\lambda$ is the security parameter.

- The access pattern of $P_{0}$ is computationally indistinguishable to that of $P_{1}$.

\section{SySTEM DETAILS}

As described previously, the basic design of ObliviSync is presented in Figure 1. In this section we highlight the implementation details further. In particular, we describe the implementation components focusing on interesting design challenges and user settings that can be used to tune the performance.

\section{A. Filesystem Description}

First, we describe the data organization of the backend files that form the storage mechanisms for the encrypted filesystem. We note that our filesystem is specifically tailored for the ObliviSync use case, and this design is what leads to our practical performance gains.

Files, fragments, blocks, block-ids. The user of an ObliviSync client is creating, writing, reading, and deleting logical files in the frontend filesystem via the FUSE mount. The ObliviSync client, to store user files, will break down the 


\section{Frontend}

file $\mathrm{X}$

\begin{tabular}{l|l|l|l|}
$x_{1}$ & $x_{2}$ & $x_{3}$ & $x_{4}$ \\
\hline
\end{tabular}

file Y

\begin{tabular}{l|l|l|}
$y_{1}$ & $y_{2}$ & $y_{3}$ \\
\hline
\end{tabular}

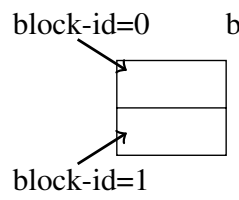

file 0

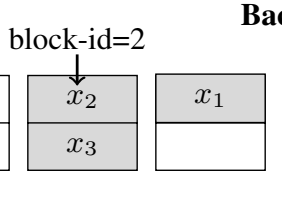

file 1

file 2
Backend

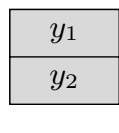

file 3

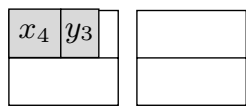

file 4 file 5
Fig. 2. An example of two front-end files stored in the backend files. The frontend file X (resp. file Y) consists of 4 (resp. 3) fragments where the last fragment is smaller than the block size. Each fragment is stored in a block. Each backend file contains exactly two blocks. Backend blocks are indexed by block-ids, starting with 0 . For example, the two blocks in file 0 has blockids 0 and 1 , respectively, and the block-ids of the blocks where fragments $x_{1}, x_{2}, x_{3}, x_{4}$ are located are $4,2,3,8$ respectively. Note the small fragments $x_{4}$ and $y_{3}$ are located in the same block with block-id 8 .

files into one or more fragments, and these fragments are then stored within various encrypted blocks in the backend.

Blocks are stored in the backend directory in block pairs of exactly two files each. (Note each block pair resides within a single file in the backend directory, but we avoid the use of the word "file" when possible to disambiguate from the logical frontend files, and instead refer to these as "block pairs".) We explain why we chose to combine exactly two blocks in each pair later when discussing performance optimization. Note that it is only these encrypted block pairs in the backend directory which are seen (and transferred) by the cloud synchronization service.

While each block has the same size, files stored in the frontend can have arbitrary sizes. A file fragment can be smaller than a block size, but not larger. In particular, each file will consist of an ordered list of fragments, only the last of which may have size smaller than that of a full block.

While files are indicated by pathnames as in any normal filesystem, backend blocks are indexed by numeric block-ids, with numbering such that the two blocks that make up a block pair are readily identified. See Figure 2 for a simple example.

Filetable, file-entries, file-ids. Since a single frontend file consists of a series of fragments (or a single fragment if the file is small) where each fragment is stored within a block, ObliviSync needs to keep track of the backend blocks that each file uses so that it may support file create/update/delete operations effectively.

For this purpose, ObliviSync maintains a filetable, consisting of file-entries. Each frontend file is one-to-one mapped to a file-entry, which maintains some file metadata and a list of block-ids in order to refer to the blocks that contain the frontend file's fragments, in order. In a sense, block-ids (resp., the file-entry) in our system are similar to position map entries (resp., the position map) in traditional ORAMs. The main difference is that in order to treat multiple front-end files, our system maintains a filetable containing multiple file-entries.
The file-entries in the filetable are indexed by file-ids. As files update, the file-id remains constant; however, based on the oblivious writing procedure, the file fragments may be placed in different backend blocks, so the block-ids may change accordingly.

Filetable B-tree. The filetable mapping file-ids to file-entries is implemented as a B-tree, with node size B proportional to the size of backend blocks. In general, the height of this B-tree is $O\left(\log _{B} n\right)$, where $n$ is the number of files stored. As we will see in Section IV-G, for typical scenarios the block size is sufficiently large so that the B-tree height can be at most 1 (i.e., the tree consists of the root node and its children), and we will assume this case for the remainder.

The leaf nodes are added and stored alongside ordinary files in blocks. There are two important differences from regular files, however: leaf nodes are always exactly the size of one full block, and they are indexed (within the root node) by their block-id directly. This way, leaf nodes have neither a file-id nor a file-entry.

Directory files. As is typical in most filesystems, file paths are grouped into directories (i.e., folders), and each directory contains the pathnames and file-ids of its contents. Observe that the directory file only changes when a file within it is created or destroyed, since file-ids are persistent between file modifications. Directory files are treated just like any other file, with the special exception that the root directory file is always assigned file-id 0 .

\section{B. Design Choices for Performance Optimization}

File-entry cache. To avoid frequent writes to the B-tree leaves, we maintain a small cache of recent file-id to file-entry mappings. Like the root node of the filetable B-tree, the size of this cache is proportional to the backend block size.

When the cache grows too large, the entries that belong in the most common leaf node among all cache entries are removed and written to that leaf node. This allows for efficient batching of leaf node updates and guarantees a significant fraction of the cache is cleared whenever a leaf node is written.

In fact, if the block size is large enough relative to the number of files, the cache alone is sufficient to store all fileentries, and the B-tree effectively has height 0 with no leaf nodes.

Superblock. Observe that every time any file is changed, its file-entry must be updated in the cache, which possibly also causes a leaf node to be written, which in turn (due to the oblivious shuffling of block-ids on writes) requires the root node of the filetable to be re-written as well.

Since the root node of the filetable B-tree and the fileentry cache are both changed on nearly every operation, these are stored specially in a single designated backend block pair called the superblock that is written on every operation and never changes location. Because this file is written every time, it is not necessary to shuffle its location obliviously.

The superblock also contains a small (fixed) amount of metadata for the filesystem parameters such as the block size and total number of backend block pairs. As mentioned above, the size of both the B-tree root node and the file-entry cache 
are set proportionally to the backend block size, so that the superblock in total has a fixed size corresponding to one block pair; our implementation stores this always in backend file 0 .

Split block: a block with small fragments. At the block level, there can be two types of blocks: a full block where the fragment stored within is as large as the block size and inhabits the entirety of the block, or a split block where multiple fragments smaller than the block size are stored within the same block. When a large file is stored as a series of fragments, we maintain that all fragments except possibly for the last fragment are stored in full blocks. That is, there will be at most one split-block fragment per file.

Introducing split blocks allows the system to use the backend storage more efficiently. For example, without using split blocks, 100 small fragments from 100 frontend files will consume 100 backend blocks, but by placing multiple small fragments into a single split block, we can reduce the number of blocks consumed.

Looking up the data in a full block is straightforward: given the block-id value, ObliviSync fetches the given block and decrypts its contents. In addition to the actual data, we also store the file-id of the file within the block itself as metadata. This will facilitate an easy check for whether a given block has become stale, as we will see shortly.

For a split block, however, the system also needs to know the location of the desired fragment within the block. The information is stored within the block itself in the block table which maps file-ids to offsets. With the size of the file from the filetable, it is straightforward to retrieve the relevant data. A full block can then be simply defined as a block without a block table, and the leading bit of each block is used to identify whether the block is full or split.

Two blocks in a backend file. All backend blocks are grouped into pairs of two consecutive blocks where each pair of blocks resides within a single backend file. Importantly, we relax slightly the indexing requirements so that small fragments are allowed to reside in either block without changing their block$i d$. This tiny block-id mismatch is easily addressed by looking up both blocks in the corresponding backend file.

Furthermore, as we will see in the sync operation described later, both blocks in a given pair are randomly selected to be repacked and rewritten at the same time. This additional degree of freedom for small fragments is crucial for bounding the worst-case performance of the system.

\section{Read-Only Client}

A read-only client (ObliviSync-RO) with access to the shared private key is able to view the contents of any directory or file in the frontend filesystem by reading (and decrypting) blocks from the backend, but cannot create, destroy, or modify any file's contents.

To perform a read operation for any file, given the file-id of that file (obtained via the directory entry), the ObliviSync-RO first needs to obtain the block-id list of the file's fragments to then decrypt the associated blocks and read the content. This is accomplished in the following steps:

1) Decrypt and read the superblock.
2) Check in the file-entry cache. If found, return the corresponding block-id list.

3) If not found in the cache, search in the filetable via the B-tree root (part of the superblock) to find the block-id of the appropriate leaf node in the B-tree.

4) Decrypt and read the leaf node to find the file-entry for the file in question, and return the corresponding block-id list and associated metadata.

Once the block-id list has been loaded, the desired bytes of the file are loaded by computing the block-id offset according to the block size, loading and decrypting the block specified by that block-id, and extracting the data in the block.

Given the file-id, it can be seen from the description above that a single read operation in ObliviSync-RO for a single fragment requires loading and decrypting at most 3 blocks from the backend: (1) the superblock, (2) a B-tree leaf node (if not found in file-entry cache), and (3) the block containing the data. In practice, we can cache recently accessed blocks (most notably, the superblock) for a short period in order to speed up subsequent lookups.

\section{Read/Write Client}

The read/write client ObliviSync-RW encompasses the same functionality as ObliviSync-RO for lookups with the added ability to create, modify, and delete files.

Pending writes buffer. The additional data structure stored in ObliviSync-RW to facilitate these write operations is the pending writes buffer of recent, un-committed changes. Specifically, this buffer stores a list of (file-id, fragment, timestamp) tuples. When the ObliviSync-RW encounters a write (or create or delete) operation, the operation is performed by adding to the buffer. For modified files that are larger than a block size, only the fragments of the file that need updating are placed in the buffer, while for smaller files, the entire file may reside in the buffer. During reads, the system first checks the buffer to see if the file-id is present and otherwise proceeds with the same read process as in the ObliviSync-RO description above. The main motivation of the buffer is to allow oblivious writing without compromising usability. The user should not be aware of the delay between when a write to a file occurs and when the corresponding data is actually synced to the cloud service provider. The function of the buffer is similar to that of "stash" in normal ORAM constructions.

Interestingly, we note that the buffer also provides considerable performance benefits, by acting as a cache for recentlyused elements. Since the buffer contents are stored in memory un-encrypted, reading file fragments from the buffer is faster than decrypting and reading data from the backend storage. The buffer serves a dual purpose in both enabling obliviousness and increasing practical efficiency.

Syncing: gradual and periodic clearing of the buffer. The buffer within ObliviSync-RW must not grow indefinitely. In our system, the buffer size is kept low through the use of a periodic sync operations wherein the buffer's contents are encrypted and stored in backend blocks.

Each sync operation is similar to a single write procedure in write-only ORAM, but instead of being triggered on each write operation, the sync operation happens on a fixed timer 


\begin{tabular}{lcccc}
\multicolumn{1}{c}{ Action } & Buffer & Backend & ObliviSync-RW view & ObliviSync-RO view \\
\hline 0. (initial) & \{\} & $\left\{f_{1}, f_{2}, f_{3}\right\}$ & {$\left[f_{1}, f_{2}, f_{3}\right]$} & {$\left[f_{1}, f_{2}, f_{3}\right]$} \\
1. Two blocks updated & $\left\{\mathbf{f}_{\mathbf{2}}^{\prime}, \mathbf{f}_{\mathbf{3}}^{\prime}\right\}$ & $\left\{f_{1}, f_{2}, f_{3}\right\}$ & {$\left[f_{1}, \mathbf{f}_{\mathbf{2}}^{\prime}, \mathbf{f}_{\mathbf{3}}^{\prime}\right]$} & {$\left[f_{1}, f_{2}, f_{3}\right]$} \\
2. One block synced & $\left\{\mathbf{f}_{\mathbf{2}}^{\prime}\right\}$ & $\left\{f_{1}, f_{2}, f_{3}, \mathbf{f}_{\mathbf{3}}^{\prime}\right\}$ & {$\left[f_{1}, \mathbf{f}_{\mathbf{2}}^{\prime}, \mathbf{f}_{\mathbf{3}}^{\prime}\right]$} & {$\left[f_{1}, f_{2}, f_{3}\right]$} \\
3. Both blocks synced & \{\} & $\left\{f_{1}, f_{2}, f_{3}, \mathbf{f}_{\mathbf{3}}^{\prime}, \mathbf{f}_{\mathbf{2}}^{\prime}\right\}$ & {$\left[f_{1}, \mathbf{f}_{\mathbf{2}}^{\prime}, \mathbf{f}_{\mathbf{3}}^{\prime}\right]$} & {$\left[f_{1}, \mathbf{f}_{\mathbf{2}}^{\prime}, \mathbf{f}_{\mathbf{3}}^{\prime}\right]$} \\
4. Stale data removed & \{\} & $\left\{f_{1}, \mathbf{f}_{\mathbf{3}}^{\prime}, \mathbf{f}_{\mathbf{2}}^{\prime}\right\}$ & {$\left[f_{1}, \mathbf{f}_{\mathbf{2}}^{\prime}, \mathbf{f}_{\mathbf{3}}^{\prime}\right]$} & {$\left[f_{1}, \mathbf{f}_{\mathbf{2}}^{\prime}, \mathbf{f}_{\mathbf{3}}^{\prime}\right]$}
\end{tabular}

Fig. 3. Example of ordering and consistency in updating two of three fragments of a single file. Use of the shadow filetable ensures that the ObliviSync-RO view is not updated until all fragments are synced to the backend.

basis. We call the time between subsequent sync operations an epoch and define this parameter as the drip time of the ObliviSync-RW.

Also, similar to the write-only ORAM, there will be a fixed set of backend files that are chosen randomly on each sync operation epoch. The number of such backend files that are rewritten and re-encrypted is defined as the drip rate. Throughout, let $k$ denote the drip rate. We discuss these parameters further and their impact on performance in Section IV-G. Overall, each sync operation proceeds as follows:

1) Choose $k$ backend block pairs randomly to rewrite and decrypt them

2) Determine which blocks in the chosen backend files are stale, i.e., containing stale fragments.

3) Re-pack the empty or stale blocks, clearing fragments from the pending writes buffer as much as possible.

4) Re-encrypt the chosen backend block pairs.

The detailed sync operation is described in the next section.

Consistency and ordering. In order to avoid inconsistency, busy wait, or race conditions, the order of operations for the sync procedure is very important. For each file fragment that is successfully cleared from the buffer into the randomly-chosen blocks, there are three changes that must occur:

1) The data for the block is physically written to the backend.

2) The fragment is removed from the buffer.

3) The filetable is updated with the new block-id for that fragment.

It is very important that these three changes occur in that order, so that there is no temporary inconsistency in the filesystem. Moreover, the ObliviSync-RW must wait until all fragments of a file have been synced before updating the fileentry for that file; otherwise there could be inconsistencies in any ObliviSync-RO clients.

The consistency is enforced in part by the use of a shadow filetable, which maintains the list of old block-ids for any files that are currently in the buffer. As long as some fragment of a file is in the buffer, the entry in the filetable that gets stored with the superblock (and therefore, the version visible to any read-only client mounts), is the most recent completely-synced version).

An example is depicted in Figure 3. Say file $f$ consists of three fragments $\left[f_{1}, f_{2}, f_{3}\right]$, all currently fully synced to the backend. Now say an ObliviSync-RW client updates the last two fragments to $f_{2}^{\prime}$ and $f_{3}^{\prime}$. It may be the case that, for example, $f_{3}^{\prime}$ is removed from the buffer into some backend block before $f_{2}^{\prime}$ is. (This could easily happen because $f_{3}^{\prime}$ is a small fragment that can be stored within a split block, whereas $f_{2}^{\prime}$ is a full block.) At this point, the shadow filetable will still store the location of $f_{3}$ and not $f_{3}^{\prime}$, so that any ObliviSync-RO clients have a consistent view of the file. It is only after all fragments of $f$ are removed from the buffer that the filetable is updated accordingly.

One consequence is that there may be some duplicate versions of the same fragment stored in the backend simultaneously, with neither being stale (as in $f_{3}$ and $f_{3}^{\prime}$ after step 2 in Figure 3). This adds a small storage overhead to the system, but the benefit is that both types of clients have a clean, consistent (though possibly temporarily outdated) view of the filesystem. Note that, even in the worst case, no non-stale fragment is ever duplicated more than once in the backend.

\section{E. Detailed Description of Buffer Syncing}

Step 1: Choosing which blocks to rewrite. As in write-only ORAM, $k$ random backend files are chosen to be rewritten at every sync operation with the following differences:

- Each backend file contains a pair of blocks, which implies that $k$ random pairs of blocks are to be rewritten.

- In addition, the backend file containing the superblock is always rewritten.

Choosing pairs of blocks together is crucial, since as we have mentioned above, small fragments are free to move between either block in a pair without changing their blockids. In addition, the superblock must be rewritten on each sync because it contains the filetable which may change whenever other content is rewritten to the backend.

Step 2: Determining staleness. Once the blocks to be rewritten are randomly selected and decrypted, the next task is to inspect the fragments within the blocks to determine which are "stale" and can be overwritten.

Tracking fragment freshness is vital to the system because of the design of write-only ORAM. As random blocks are written at each stage, modified fragments are written to new blocks, and the file-entry is updated accordingly, but the stale data fragment is not rewritten and will persist in the old block because that old block may not have been selected in this current sync procedure. Efficiently identifying which fragments are stale becomes crucial to clearing the buffer.

A natural, but flawed, solution to tracking stale fragments is to maintain a bit in each block to mark which fragments are fresh or stale. This solution cannot be achieved for the same reason that stale data cannot be immediately deleted updating blocks that are not selected in the sync procedure are not possible. 
Instead, recall from the block design that each block also stores the file-id for each fragment. To identify a stale fragment, the sync procedure looks up each fragment's file-id to get its block-id list. If the current block's identifier is not in the block-id list, then that fragment must be stale.

Step 3: Re-packing the blocks. Then next step after identifying blocks and fragments within those blocks that are stale (or empty) is to re-pack the block with the non-stale fragments residing within the block and fragments from the buffer.

One important aspect to consider when re-packing the blocks is to address the fragmentation problem, that is, to reduce the number of blocks that small fragments use so that there remain a sufficient number of blocks for full-block fragments.

A naïve approach would be to evict all the non-stale fragments from the selected blocks and consider all the fragments in the buffer and the evicted fragments to re-pack the selected blocks with the least amount of internal fragmentation. While this would be a reasonable protocol for some file systems to reduce fragmentation, this would require (potentially) changing all of the file-entries (in particular, the block-id list) for all fragments within the selected blocks. That would be problematic because it is precisely these old entries which are likely not to be in the file-entry cache, and therefore doing this protocol would require potentially changing many filetable B-tree nodes at each step, something that should be avoided as writes are expensive.

Instead, we take a different approach in order to address the fragmentation problem and minimize the block-id updates for the non-stale fragments at the same time. Our approach has two stages: the placement of non-stale fragments and then clearing the fragments in the buffer.

Placement of non-stale fragments. We use the following rule when addressing the existing non-stale fragments.

Non-stale full-block fragments stay as they are, but non-stale small fragments may move to the other block in the same backend file.

Recall that blocks are paired in order and share a single backend file, and so this flexibility enables small fragments to be re-packed across two blocks to reduce fragmentation without having to update the block-id value. Further, this solution also avoids a "full-block starvation" issue in which all blocks contained just a small split-block fragment. After re-packing, the small fragments in each block pair may be combined into a single split block, leaving the other block in the pair empty and ready to store full block fragments from the buffer. In other words, the re-pack procedure ensures that existing full-block fragments do not move, but existing small-block fragments are packed efficiently within one of the blocks in a pair to leave (potentially) more fully empty blocks available to be rewritten.

Pushing fresh fragments. At this point, the randomly-chosen blocks are each either: (a) empty, (b) filled with an existing full-block fragment, or (c) partially filled with some small fragments. The block re-packing first considers any directory file fragments in the buffer, followed by any regular file fragments, each in FIFO order. (Giving priority status to directory files is important to maintain low latency, as discussed in the next section.) The synchronization process then proceeds the same for all fragments in the buffer: for each fragment, it tries to pack it into the randomly-selected blocks as follows:

- If it is a full-block fragment, it is placed in the first available empty block (case (a)), if any remain.

- If it is a small fragment, it is placed if possible in the first available split block (case (c)) where there is sufficient room.

- If it is a small fragment but it cannot fit in any existing split block, then the first available empty block (case (a)), if any, is initialized as a new split block containing just that fragment.

In this way, every buffer fragment is considered for repacking in order of age, but not all may actually be re-packed. Those that are re-packed will be removed from the buffer and their filetable entries will be updated according to the chosen block's block-id.

A key observation that will be important in our runtime proof later is that after re-packing, either (1) the buffer is completely cleared, or (2) all the chosen blocks are nonempty.

Step 4: Re-encrypting the chosen backend files. After the re-packing is complete, the sync procedure re-encrypts the superblock (which always goes at index 0), as well as all the re-packed blocks, and stages them for writing back to backend files. The actual writing is done all at once, on the timer, immediately before the next sync operations, so as not to reveal how long each sync procedure took to complete.

\section{F. Frontend FUSE Mounts}

The FUSE (file system in user space) mounts are the primary entry point for all user applications. FUSE enables the capture of system calls associated with I/O, and for those calls to be handled by an identified process. The result is that a generic file system mount is presented to the user, but all accesses to that file system are handled by either the ObliviSync-RW or ObliviSync-RO client that is running in the background.

The key operations that are captured by the FUSE mount and translated into ObliviSync-RW or ObliviSync-RO calls are as follows:

- create (filename): create a new (empty) file in the system in two steps. First a new file-id is chosen, and the corresponding file-entry is added to the filetable. Then that file-id is also stored within the parent directory file.

- delete(filename): remove a file from the system by removing it from the current directory file and removing the associated file-entry from the filetable.

- read(filename, offset, length) $\rightarrow$ data : read data from the identified file by looking up its file-id in the current directory and requesting the backend ObliviSync-RW or ObliviSync-RO to perform a read operation over the appropriate blocks.

- write (filename, of $f$ set, length) : write data to the identified file by looking up its file-id in the current directory and then adding the corresponding fragment(s) to the ObliviSync-RW's buffer for eventual syncing. 
- resize(filename, size) : change the size of a file by looking up its file-entry in the current directory and changing the associated metadata. This may also add or remove entries from the corresponding block-id list if the given size represents a change in the number of blocks for that file. Any added blocks will have negative block-id values to indicate that the data is not yet available.

Of course, there are more system calls for files than these, such as open() or stat () that are implemented within the FUSE mount, but these functions succinctly encompass all major operations between the frontend FUSE mount and backend file system maintenance.

As noted before, the FUSE mount also maintains the file system's directory structure whose main purpose is to link file names to their file-id values, as well as store other expected file statistics. The directory files are themselves treated just like any other file, except that (1) the root directory always has file-id 0 so it can be found on a fresh (re-)mount, and (2) directory files are given priority when syncing to the backend.

For a file to become available to the user, it must both be present in the backend and have an entry in the directory file. Without prioritization of directory files, it may be the case that some file is available without the directory entry update, thus delaying access to the user. Conversely, the opposite can also be true: the directory entry shows a file that is not completely synchronized. Fortunately, this situation is easy to detect upon open () and an IO-error can be returned which should be handled already by application making the system call.

The FUSE module is aware of some backend settings to improve performance, notably the block size. When a file is modified, it is tracked by the FUSE module, but for large files, with knowledge of the block size, the FUSE module can identify which full fragments of that file are modified and which remain unchanged. Only the fragments with actual changes are inserted into the buffer to be re-packed and synced to the backend.

\section{G. Key parameter settings}

The tunable parameters for a ObliviSync implementation consist of:

- $B$ : the size of each backend file (i.e., block pair)

- $N$ : the total number of backend files

- $n$ : the total number of frontend files (i.e., logical files)

- $t$ : the drip time

- $k$ : the drip rate

Backend files. The first two parameters $B$ and $N$ depend on the backend cloud service. A typical example of such parameters can be taken from the popular Dropbox service, which optimally handles data in files of size $4 \mathrm{MB}$, so that $B=2^{22}[11]$, and the maximal total storage for a paid "Dropbox Pro" account is 1TB, meaning $N=2^{18}$ files would be the limit. Note that, as our construction always writes blocks in pairs, each block pair is stored in a single file and the block size in ObliviSync will be $B / 2$.

Frontend files. The next parameter $n$ is not really a parameter per se but rather a limitation, as our construction requires $n \leq$ $B^{2}$ in order to ensure the filetable's B-tree has height at most
1. For $B=2^{22}$, this means the user is "limited" to roughly 16 trillion files.

Drip time and drip rate. The drip time and drip rate are important parameters for the buffer syncing. The drip time is the length of the epoch, i.e., the time between two consecutive syncs to the backend. The drip rate refers to how many block pairs are randomly selected for rewriting on each epoch.

These two parameters provide a trade-off between latency and throughput. Given a fixed bandwidth limitation of, say, $x$ bytes per second, $(k+1) B$ bytes will be written every $t$ seconds for $k$ randomly chosen backend files and the superblock, so that we must have $(k+1) B / t \leq x$. Increasing the drip time and drip rate will increase latency (the delay between a write in the ObliviSync-RW appearing to ObliviSync-RO clients), but it will increase throughput as the constant overhead of syncing the superblock happens less frequently.

We will consider in our experimentation section (see Section VI) the throughput, latency, and buffer size of the system under various drip rate and drip time choices. Our experiment indicates that for most uses, the smallest possible drip time $t$ that allows a drip rate of $k \geq 3$ files per epoch should be chosen.

\section{ANALYSIS}

\section{A. Time to write all files}

In this subsection we will prove the main Theorem 1 that shows the relationship between the number of sync operations, the drip rate, and the size of the buffer. Recall from the preceding subsection the parameters $B$ (block pair size), $N$ (number of backend block pairs), and $k$ (drip rate). Specifically, we will show that, with high probability, a buffer with size $s$ is completely cleared and synced to the backend after $O\left(\frac{s}{B k}\right)$ sync operations. This is optimal up to constant factors, since only $B k$ bytes are actually written during each sync.

Theorem 1. For a running ObliviSync-RW client with parameters $B, N, k$ as above, let $m$ be the total size (in bytes) of all non-stale data currently stored in the backend, and let $s$ be the total size (in bytes) of pending write operations in the buffer, and suppose that $m+s \leq N B / 4$.

Then the expected number of sync operations until the buffer is entirely cleared is at most $4 s /(B k)$.

Moreover, the probability that the buffer is not entirely cleared after at least $\frac{48 s}{B k}+18 r$ sync operations is at most $\exp (-r)$.

Before giving the proof, let us summarize what the this theorem means specifically.

First, the condition $m+s \leq N B / 4$ means that the guarantees hold only when at most $25 \%$ of the total backend capacity is utilized. For example, if using Dropbox with 1TB of available storage, the user should store at most 250GB of files in the frontend filesystem in order to guarantee the performance specified in Theorem 1 .

Second, as mentioned already, the expected number of sync operations is optimal (up to constant factors), as the total amount of data written in the frontend cannot possibly exceed the amount of data being written to the backend. 
In the number of syncs $48 s /(B k)+18 r$ required to clear the buffer with high probability, one can think of the parameter $r$ as the number of "extra" sync operations required to be very sure that the buffer is cleared. In practice, $r$ will be set proportionally to the security parameter. A benefit of our construction compared to many other ORAM schemes is that the performance degradation in terms of the security parameter is additive and not multiplicative. Put another way, if it takes 1 extra minute of syncing, after all operations are complete, in order to ensure high security, that extra minute is fixed regardless of how long the ObliviSync-RW has been running or how much total data has been written.

Finally, a key observation of this theorem is that it does not depend on the distribution of file sizes stored in the frontend filesystem, or their access patterns, but only the total size of data being stored. The performance guarantees of our system therefore allow arbitrary workloads by the user, provided they can tolerate a constant-factor increase in the backend storage size.

We now proceed with the proof of Theorem 1 .

Proof: There are $N$ blocks of backend storage. Each stores some combination of at most two split blocks and full blocks. Full blocks have size $\frac{B}{2}$ each, and split blocks contain multiple fragments summing to size at most $\frac{B}{2}$ each.

Suppose some sync operation occurs (selecting $k$ block pairs from the backend, removing stale data and re-packing with new fragments from the buffer), and afterwards the buffer is still not empty. Then it must be that case that the $k$ block pairs that were written are at least half filled, i.e., their total size is now at least $\frac{k B}{2}$. The reason is, if any block pair had size less than $\frac{B}{2}$, then it could have fit something more (either a full block or a fragment) from the buffer. But since the buffer was not emptied, there were no entirely empty blocks among the $k$ block pairs.

Furthermore, because $m<\frac{N B}{4}$ while the buffer is not empty, the expected size of a single, randomly-chosen pair of blocks is less than $\frac{B}{4}$. By linearity of expectation, the expected size of $\mathrm{k}$ randomly selected block pairs is less than $\frac{k B}{4}$.

Combining the conclusions from the preceding paragraphs we see that, on any sync operation that does not empty the buffer completely, the $k$ randomly selected block pairs go from expected size less than $\frac{k B}{4}$, to guaranteed size greater than $\frac{k B}{2}$. This means the expected decrease in buffer size in each sync is at least $\frac{k B}{4}$. Starting with $\mathrm{s}$ bytes in the buffer, the expected number of syncs is therefore less than $\frac{s}{k B / 4}=\frac{4 s}{B k}$.

Now we extend this argument to get a tail bound on the probability that the buffer is not emptied after $T=48 s /(B k)+$ $18 r$ sync operations, for some $r \geq 0$.

Call a sync operation productive if it results in either the buffer being cleared entirely, or the size of the buffer decreasing by at least $\frac{k B}{8}$. And define $Y$ to be a random variable for the total size of the $k$ randomly-selected blocks for a single operation.

From above, a sync is guaranteed to be productive whenever $Y \leq \frac{3 k B}{8}$ (because the total size after the sync will be at least $\frac{k B}{2}$ ). We also know from above that $\mathbb{E}[Y]=\frac{k B}{4}$. Using the Markov inequality, we have
$\operatorname{Pr}\left[Y \leq \frac{3 k B}{8}\right]=1-\operatorname{Pr}\left[Y>\frac{3 k B}{8}\right] \geq 1-\frac{k B / 4}{3 k B / 8}=\frac{1}{3}$

That is, each sync is productive with probability at least $\frac{1}{3}$.

Next, define a random variable $X$ to be the number of productive syncs among a series of $T=48 s /(B k)+18 r$ sync operations. Importantly, if $X \geq 8 s /(B k)$, then the buffer will be cleared at the end of $T$ syncs.

We see that $X$ is the sum of $T$ i.i.d. Bernoulli trials, each with probability $p=\frac{1}{3}$. Therefore the Hoeffding bound from [16] tells us that, for any $\epsilon>0$,

$$
\operatorname{Pr}\left[X<\left(\frac{1}{3}-\epsilon\right) T\right] \leq \exp \left(-2 \epsilon^{2} T\right) .
$$

Setting $\epsilon=\frac{1}{6}$ works to bound the probability that $X<$ $8 s /(B k)$, since $\frac{8 s}{B k}<\frac{1}{6}\left(\frac{48 s}{B k}+18 r\right)$ for any $r>0$. The theorem follows from $\exp \left(-2 \epsilon^{2} T\right)=\exp \left(-\frac{1}{18} \cdot\left(\frac{48 s}{B k}+18 r\right)\right)<$ $\exp (-r)$.

\section{B. Security}

Theorem 2. Let $\lambda$ be the security parameter. Consider ObliviSync-RW with parameters $B, N, k$ as above, and with drip time $t$. For any $L$ and $t$ as fsequence parameters, ObliviSync-RW is strongly-secure write-only filesystem with running time $T=t+\frac{48 L t}{B k}+18 \lambda t$.

Proof: We need to show the following:

- ObliviSync-RW finishes all tasks in each sequence within time $\mathrm{T}$ with probability $1-n e g(\lambda)$.

- For any two $(L, t)$-fsequences $P_{0}$ and $P_{1}$, both access patterns are computationally indistinguishable from each other.

The first condition is achieved according to Theorem 1, since one sync operation occurs every $t$ seconds.

It is left to show that the second condition also holds. Obliviousness mostly follows from the original write-only ORAM security. To achieve strong obliviousness, we stress that ObliviSync-RW always writes encrypted data in $k$ files at the backend chosen independently and uniformly at random. In particular:

- If there is too much data to be synchronized, the remaining data is safely stored in the temporary buffer so that the data will be eventually synchronized. Theorem 1 makes sure this must happen with overwhelming probability.

- If there is too little (or even no) data to be synchronized, the system generates what amounts to dummy traffic (repacking the $k$ chosen block pairs with the same data they stored before).

Therefore, the second condition is also satisfied.

There is an important assumption in both theorems above, namely that the client is actually able to execute the sync operation with the given drip rate $k$ within each epoch with drip time $t$. If the parameters $k$ and $t$ are set too aggressively, it may be the case that the sync operation takes longer than $t$ seconds to complete, and this fact would be noticed by the 
cloud server or indeed any network observer. While the leakage in such cases is relatively minor (an indication that, perhaps, the client's system load is higher than normal), it is nonetheless important for security to ensure $t$ and $k$ are set appropriately to avoid this situation of a sync operation "blowing through" the epoch.

\section{EXPERIMENTS}

We fully implemented ObliviSync using python3 and fusepy [2], and the source code of our implementation is available on GitHub as well as a video demonstration [14] and a Dockerfile for quick setup and testing.

To evaluate ObliviSync, we performed a series of experiments to test its performance at the extremes. In particular, we are interested in the following properties:

- Throughput with fixed-size files: If the user of ObliviSync were to insert a large number of files all at once, the buffer will immediately be filled to hold all the insertions. How long does it take (in number of epochs) for each of the files to sync to the read end?

- Throughput with variable-size files: If the users were to insert a large number of variable size files all at once, instead of fixed-sized ones, how does the throughput change? This experiment will verify Theorem 1, which states that the performance of our system depends on the total number of bytes (instead of the total number of files) in the pending write buffer.

- Latency: If the user of ObliviSync-RW were to insert a large number of files one at a time, how long does it take for each of the files to appear to a different ObliviSync-RO user?

- The size of pending writes buffer: We also investigate how much space the pending writes buffer uses while the system is working under different loads with realistic file sizes. Recall the pending writes buffer works similarly as the stash in the write-only ORAM, and it is important that this buffer does not grow too large.

- Functionality with Dropbox backend: Finally, we performed throughput and latency experiments with Dropbox as the backend storage mechanism for ObliviSync, and compare its performance to that of EncFS [13] on Dropbox. EncFS is a per-file encrypted file system tool that provides no obliviousness.

\section{A. Throughput with Fixed-size Files}

We first consider the throughput in our system. In particular, we are interested in the performance as it relates to the availability of backend blocks.

To limit the factors in these experiments, we use the following parameters:

- $N=1024$; we used 1024 backend files (i.e., block pairs).

- $n=920$; we attempted to insert 920 frontend files (or $90 \%$ full).

- $B=1 \mathrm{MB}$; each backend file is $1 \mathrm{MB}$ in size.

In our experiments, each frontend file is $1 \mathrm{MB}$ and thus fills up two full blocks (including any metadata). There is also two

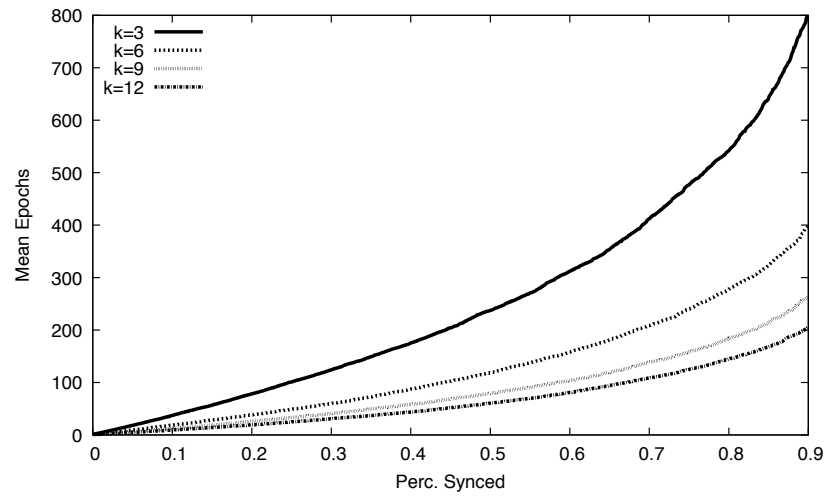

Fig. 4. Throughput for different drip rates. We used 1024 backend files, each with $1 \mathrm{MB}$, and attempted to insert 920 frontend files all at once, where each frontend file is also $1 \mathrm{MB}$. The results are the mean of three runs. With drip rate 3 (the solid line for $k=3$ ), it takes about 120 epochs on average to sync $25 \%$ of the frontend files. In addition, the graph shows the situation shifts as the backend files become more full and it becomes harder to clear the buffer.

additional block pairs in the system for the superblock and the directory entry. Overall, the entire backend storage for the system was $N \cdot B=1 \mathrm{~GB}$.

In the throughput experiment, we established an empty ObliviSync-RW and attached an ObliviSync-RO to the backend. We then wrote 920 two-block size files all at once to the ObliviSync-RW FUSE mounted frontend. We then manually called the synchronize operation that performs the oblivious writing to the backend. By monitoring the ObliviSync-RO FUSE mounted frontend, we can measure how many epochs are required to fully synchronize all the files.

Bandwidth overhead: $\mathbf{2 x}$ until $\mathbf{2 5 \%}$ of the load. In Figure 4, we graph the number of epochs (i.e., the number of timed sync operations) it takes for that percentage of files to synchronize and appear at the read-end. We conducted the experiments for different drip rates $(k)$, i.e., the number of backend files randomly selected at each epoch for writing. The results presented are the average of three runs for drip rates set to $3,6,9$, and 12 .

As one interesting data point, the graph shows that with drip rate 3 (the solid line for $k=3$ ), it takes about $\sim 120$ epochs on average to sync $25 \%$ of the frontend files. Note that the number of bytes that would be transferred to the cloud storage during 120 epochs is $120 \cdot(k+1) \cdot B=480$ $\mathrm{MB}$, and $25 \%$ of the frontend files amounts to $250 \mathrm{MB}$. So, the experiment shows that the system needs only $2 x$ bandwidth overhead, when the front-end files occupies at most $25 \%$ of the total cloud storage, with the parameters chosen in this experiment. This is better performance than what is shown in Thoerem 1 , which provably guarantees $4 \mathrm{x}$ bandwidth overhead.

Linear costs until $33 \%$ of the load. Looking closely at the graph, particularly $k=3$ trend-line, there are two regimes: linear and super-linear synchronization. In the linear regime there are enough empty blocks that on each epoch, progress is very likely to be made by clearing files from the buffer and writing new blocks to the backend. In the super-linear regime, however, there are no longer enough empty blocks to reliably clear fragments from the buffer. For $k=3$, this 


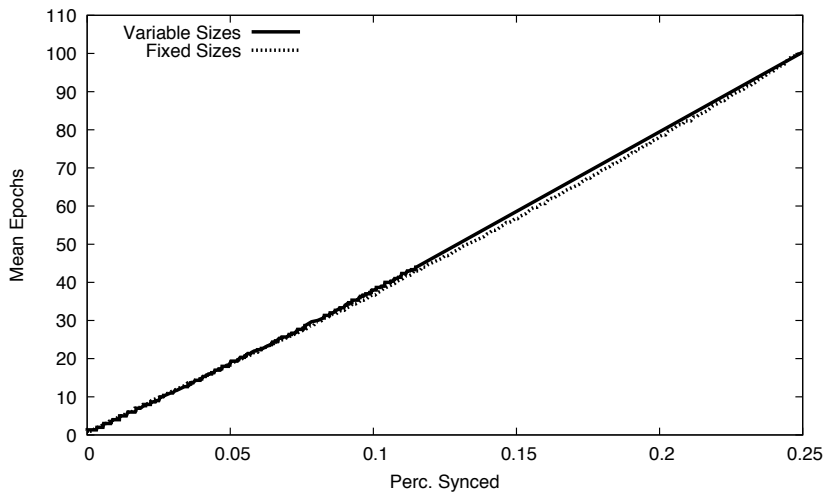

Fig. 5. Comparing throughput of inserting realistic workload of variable sizes files to the same sized insert of fixed size files. The experiment were performed with parameters $N=1024, B=1 \mathrm{MB}, k=3$. In the set of fixed-size files, each frontend file is $1 \mathrm{MB}$. There are 4,179 files in the set of variable-size files. Both set is $250 \mathrm{MB}$ in total. For both sets, the system takes about 100 epochs to sync the $250 \mathrm{MB}$ of frontend data.

regime seems to take over around $40 \% \sim 60 \%$, and the trendline's slope begins to increase dramatically. This is because each additional block written further exacerbates the problem, so it takes an increasing amount of time to find the next empty block to clear the buffer further.

The inflection point, between linear and super-linear, is particularly interesting. Apparent immediately is the fact that the inflection point is well beyond the $25 \%$ theoretic bound; even for a drip rate of $k=3$, it manages to get at least $1 / 3$ full before super-linear tendencies take over. Further, notice that for higher drip rates, the inflection point occurs for higher percentage of fullness for the backend. This is to be expected; if you are selecting more blocks per epoch, you are more likely to find an empty block to clear the buffer. But we hasten to point out that there is a trade-off in practice here.

\section{B. Throughput with Variable-size Files}

As mentioned above, an important performance property of ObliviSync is that the rate of synchronization is dependent on the total number of bytes in the pending write buffer and the fullness of the backend blocks: it does not depend on the sizes of the individual files.

To show this property clearly, we performed a similar throughput experiment as described previously, i.e., with $N=$ $1024, B=1 \mathrm{MB}$, and $k=3$, except we inserted variable size files that are drawn from realistic file distributions [10], [25]:

- The variable size files, in total, were $0.25 \mathrm{~GB}$, the same size as the fixed size files.

- The files contained 4,179 files, much larger than 250 for the case of fixed-size files.

- Interestingly, one of the variable size files was significantly larger, $144 \mathrm{MB}$, roughly the length of a short, TVepisode video.

As in the prior throughput experiment, we connected an ObliviSync-RW and ObliviSync-RO to a shared backend directory. We loaded the file set completely into the ObliviSync-RW, and then counted how many epochs it takes for the data to appear in the ObliviSync-RO FUSE file.

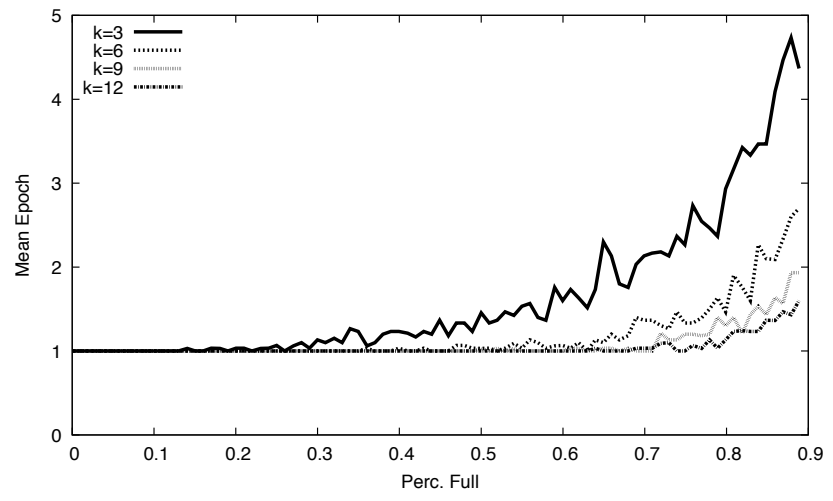

Fig. 6. Latency for different drip rates. The experiment were performed with parameters $N=1024, B=1 \mathrm{MB}$. The drip rates $k$ varies with $k=$ $3,6,9,12$. The 920 frontend files, each with $1 \mathrm{MB}$, were written one after another. For example, the point $(0.7,2)$ of the solid line with $k=3$ means that once 700 files have been written, it took about 2 epochs to sync the 701 st file.

Good performance for variable-size files. The primary result is presented in Figure 5. The two trend-lines are nearly identical, and in fact, after three runs, the average number of epochs needed to synchronize the two file loads is the same, 100 epochs. This clearly shows that our systems is dependent on the total number of bytes to synchronize and not the size of the individual files.

\section{Latency}

In this experiment, we are interested in the latency of our system. As before, we performed the experiment with $N=1024$ and $B=1 \mathrm{MB}$, and we had ObliviSync-RW and ObliviSync-RO clients with a shared backend, writing to ObliviSync-RW FUSE mount and measuring synchronization rate to the ObliviSync-RO FUSE mount. To measure latency, we only add one frontend file at a time. For example, the second frontend file gets written right after the first frontend file is completely synced. We measured how long it took for each file to synchronize in terms of the number of manual synchronizations (or epochs) required. Again, we varied the drip rate.

About 1 epoch to sync, even for high fill rates. The results in Figure 6 are the average of three runs in each drip rate. Again, there are two general regimes to the graph, a linear one and a super-linear one, and the transitions between them are, again, better than our theoretic $25 \%$ bound. First, for lower fill rates, the time to complete a single file synchronization is roughly one epoch.

At higher fill rates, it starts to take more epochs, on average, to sync a single file; however, even for the most conservative $k=3$, it only takes at most 5 epochs even for very high fill rates. For more aggressive drip rates, $k=9,12$ the impact of higher filler rates is diminished, still only requiring about 2 epochs to synchronize a single file. This is to be expected as selecting more backend files for writing increased the likelihood of finding space to clear the buffer. 


\section{The Size of Pending Writes Buffer}

In this experiment, we investigate how much space the pending writes buffer requires while the system is working. To do so, we consider more realistic file sizes and file write patterns under high thrashing rates, contrary to most of the previous experiments where each frontend file has two fullblock fragments.

We inserted frontend files of varied size based on known file size distributions such that the backend was filled to $20 \%$, $50 \%$, or $75 \%$. The file sizes were based on prior published work in measuring file size distributions. In particular, we followed a lognormal distribution, which has been shown to closely match real file sizes [10], fit with data from a largescale study of file sizes used in a university setting [25]. The same distribution was used in the variable file size experiment previously.

We also generated a series of writes to these files such that, on average, $1 \mathrm{MB}$ of data was updated on each write. This could occur within a single file or across multiple files. We selected which file to thrash based on distributions of actual write operations in the same study [25], used to generate the original file sizes. Roughly, this distribution gives a stronger preference to rewriting smaller files. We did not write exactly $1 \mathrm{MB}$ of data in each batch, but rather kept the average of each batch size as close to $1 \mathrm{MB}$ as possible in accordance with the experimental write size distribution. In particular, there were batches were a file larger than $1 \mathrm{MB}$ was written. As before, we used $N=1024$ and $B=1 \mathrm{MB}$. We used the drip rate $k=3$ to show the most conservative setting of ObliviSync. We averaged the results of three independent runs.

Reasonable buffer size: at most $2 \mathrm{MB}$. The primary result is presented in Figure 7 where we measure the number of bytes in the buffer on each synchronization. In the graph, the point $(x, y)$ means for $y$ fraction of observed execution time, the size of the buffer was greater than $x$. For example, when the backend is filled with $20 \%$ (the solid line), only for 0.2 fraction of the observed execution time, the buffer size is roughly greater than $2^{18}$ bytes. In addition, the buffer size is always larger than $2^{15}$, and the buffer never grows larger than about $2 M B$, which corresponds to only 4 blocks.

Clearly, as the fill rate increases, the amount of uncommitted data in the buffer increases; however, the relationship is not strictly linear. For example, with $20 \%$ full and $50 \%$ full, we see only a small difference in the buffer size for this extreme thrashing rate. The synchronization is able to keep up with the high thrashing rate for two main reasons: first, on each synchronization, it is generally able to clear something out of the buffer; and second, some writes occur on the same files and on small files (as would be the case in a real file system), which allows these writes to occur on cached copies in the buffer and the smaller files to packed together efficiently into blocks, even partially full ones.

At a fill rate of $75 \%$, however, there is a noticeable performance degradation. Because most of the blocks selected at each epoch are either full or do not have enough space, due to fragmentation, the buffer cannot always be cleared at a rate sufficient to keep up with incoming writes. Thus, the size of the buffer doubles in comparison with the other workloads.

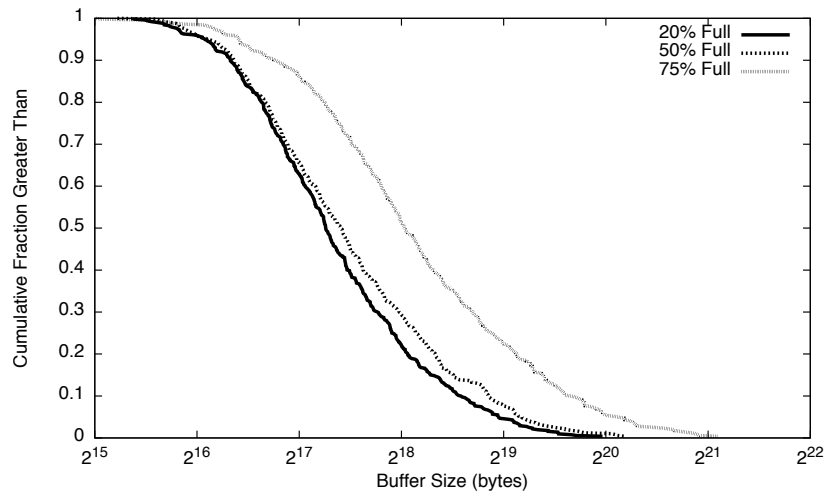

Fig. 7. Buffer size under realistic file distributions. The experiment was performed with parameters $N=1024, B=1 \mathrm{MB}, k=3$. The point $(x, y)$ means for $y$ fraction of observed execution time, the size of the buffer was greater than $x$. For example, when the backend is filled with $20 \%$ (the solid line with $20 \%$ full), only for 0.2 fraction of the entire execution time, the buffer size is roughly greater than $2^{18}$ bytes. In addition, the buffer size is always larger than $2^{15}$.

\section{E. Measurements on Dropbox}

Here, we measure the performance of ObliviSync on a real cloud synchronization service, namely Dropbox.

We performed both a latency and throughput measurement, just as before, using $1 \mathrm{MB}$ backend files, but this time the backend directory was stored within a Dropbox synchronized folder with measurements taken across two computers on our institution's network that had access to the synchronized folder.

In these experiments, we used a drip rate of $k=3$ and a drip time $t=10$ seconds. We experimented with lower drip times, but found that due to rate limiting of the Dropbox daemon on the synchronized folders, a longer drip time is required in order to to ensure that we do not blow through the epoch boundary.

In both the latency and throughout experiments described below, we established a dropbox connection on two computers on our institution's network. We designated one computer as the writer and one the reader. On the writer, it ran ObliviSync-RW and ObliviSync-RO, on a shared backend folder stored within the Dropbox synchronization folder. The writer computer measured the amount of time it took for the ObliviSync-RW to synchronize to the local ObliviSync-RO mount. Meanwhile, the reader computer ran a ObliviSync-RO mount only and monitored the file systems for the appearance of synchronized files. The difference between the ObliviSync-RO mount on the write computer and the ObliviSync-RO mount on the read computer is the propagation delay imposed by Dropbox. Additionally, as we do not have insight into how the Dropbox daemon chooses to synchronize, it is also possible that other factors are coming into play, such as taking incremental snapshots of deleted files.

Baseline: EncFS. Additionally, we wish to provide a baseline comparison of the overhead of ObliviSync, and so we performed similar experiments using EncFS [13] as the data protection mechanism. Much like ObliviSync, EncFS using a FUSE mount to display an encrypted file system that is stored in a backend folder, but EncFS provides no oblivious protection. Instead files are simply stored individually encrypted, so 


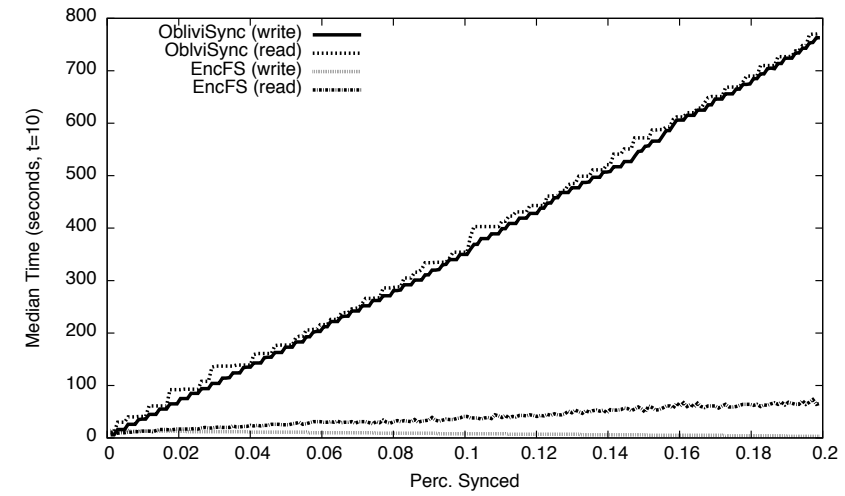

Fig. 8. Throughput of writing $0.25 \mathrm{~GB}$ of $1 \mathrm{MB}$ files all at once for ObliviSync and EncFS on Dropbox synchronized backend between two machines running on the same network. We used a drip time of 10 seconds for ObliviSync $(t=10)$ and a drip rate of $3(k=3)$ for a conservative estimate.

the total number of files is revealed as well as their sizes and full access patterns.

Throughput over Dropbox. The throughput measurement occurred much like as described earlier in the section. For both EncFS and ObliviSync, we inserted a large number of files, namely $20 \%$ full or $\sim 200 \mathrm{MB}$, and then we measured how long it takes for the buffer to clear and all files to become available. Like before, we used a read and write computer, and we measured the difference in the local and remote propagation delays of file synchronization. The primary result is presented in Figure 8.

For EncFS on the write computer, the propogation delay for all the files is nominal with files appearing nearly immediately. On the read computer, there is a propagation delay associated with Dropbox remote synchronization, and all files are accessible within 100 seconds. For ObliviSync on the write computer, we see a very similar throughput trend-line as in the prior experiments. In total, it takes just under 800 seconds (or 80 epochs) for all the files to synchronize. Interestingly, on the read computer, the propagation delay is relatively small, with respect to the overall delay, and files are accessible within an additional epoch or two. In total, these results clearly demonstrate that ObliviSync is functional and efficient to use over cloud synchronization services like Dropbox.

Latency over Dropbox. Figure 9 shows the primary result of running ObliviSync and EncFS using Dropbox as the cloud synchronization service. The EncFS write line is nearly 0 (s) as immediately upon writing the file it becomes available to write computer. However on the read computer, it takes a little under 5 seconds for the synchronization with Dropbox to complete for the same file to be accessible. This measurement forms a baseline of performance for the rate of DropBox synchronization without ObliviSync.

For ObliviSync, on the write computer, we see an expected performance metric of just under 10 seconds for each file to be visible to the read mount. The reason it is under 10 seconds and not exactly 10 seconds, as the setting of the drip time, is that a write occurring between epoch timers will take less than an epoch to sync. The propagation rate to the read computer takes a similar time as that of EncFS ( $\sim 5$ seconds); however, there is higher variance as more files need to be transferred

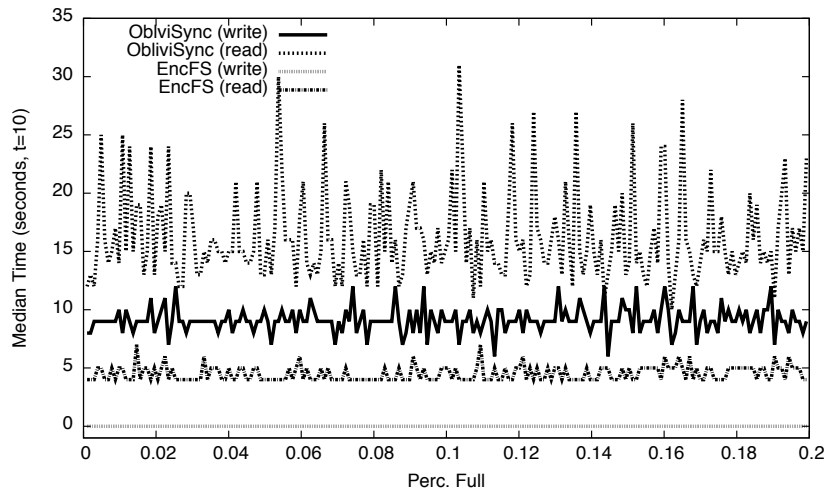

Fig. 9. Latency of writing $1 \mathrm{MB}$ files one at a time for ObliviSync and EncFS on Dropbox synchronized backend between two machines running on the same network. We used a drip time of 10 seconds for ObliviSync $(t=10)$ and a drip rate of $3(k=3)$ for a conservative estimate. The variations in the trend lines are likely due to jigger in the network.

by the Dropbox service per epoch (namely $4=k+1$ with the superblock). Still, this added variance is within $3 \mathrm{x}$ in terms of epochs: it takes at most 30 seconds for a file to sync (or 3 epochs of waiting), which is very reasonable considering the built-in overhead of the system.

\section{RELATED WORK}

ORAM. ORAM protects the access pattern from an observer such that it is impossible to determine which operation is occurring, and on which item. The seminal work on the topic is by Goldreich and Ostrovsky [15], and since then, many works have focused on improving efficiency of ORAM in both the space, time, and communication cost complexities (for example [22], [18], [23], [17], [19] just to name a few; see the references therein). Blass et al. introduced write-only ORAMs [8]. In a write-only ORAM, any two write accesses are indistinguishable, and they achieved a write-only ORAM with optimal $O(1)$ communication complexity and only polylogarithmic user memory. Based on their work, we construct a write-only ORAM that additionally supports variable-size data and hides the when the data items are modified. We point out also that variable-sized blocks in traditional read/write ORAMs were also considered recently by [21], but with higher overhead than what can be achieved in the write-only setting.

Protecting against timing side-channels. Side-channel attacks that use network traffic analysis in order to learn private information have been considered in contexts other than secure cloud storage. Proposed systems for location tracking [20] and system logging [9] use buffering and random or structured delays to protect against such attacks in a similar way to our work.

Personal cloud storage. A personal cloud storage offers automatic backup, file synchronization, sharing and remote accessibility across a multitude of devices and operating systems. Among the popular personal cloud storages are Dropbox, Google Drive, Box, and One Drive. However, privacy of cloud data is a growing concern, and to address this issue, many personal cloud services with better privacy appeared. Among the notable services are SpiderOak [5], Tresorit [6], Viivo [7], BoxCryptor [1], Sookas [4], PanBox [3], and OmniShare [24]. 
All the solutions achieve better privacy by encrypting the file data using encryption keys created by the client. We stress that however there has been no attempt to achieve the stronger privacy guarantee of obliviousness.

\section{CONCLUSION}

In this paper, we report our design, implementation, and evaluation of ObliviSync, which provides oblivious synchronization and backup for the cloud environment. Based on the key observation that for many cloud backup systems, such as Dropbox, only the writes to files are revealed to cloud provider while reads occur locally, we built upon write-only ORAM principles such that we can perform oblivious synchronization and backup while also incorporating protection against timing channel attacks. When the drip-rate and drip time parameters are set properly according to the usage pattern, this overhead is just $4 \mathrm{x}$ both in theory and in practice.

We also consider practicality and usability. ObliviSync is designed to seamlessly integrate with existing cloud services, by storing encrypted blocks in a normal directory as its backend. The backend can then be stored within any cloud based synchronization folder, such as a user's Dropbox folder. To be stored within the backend encrypted blocks, we designed a specialized block-based file system that can handle variable size files. The file system is presented to the user in a natural way via a frontend FUSE mount such that the userfacing interface is simply a folder, similar to other cloud synchronization services. Any modifications in the frontend FUSE mount are transparently and automatically synchronized to the backend without leaking anything about the actual writes that have occurred.

In evaluating our system, we can prove that the performance guarantees hold when $25 \%$ of the capacity of the backend is used, and our experimental results find that, with realistic workloads, much higher capacities can in fact be tolerated while maintaining very reasonable efficiency. Importantly, ObliviSync can be tuned to the desired application based on modifying the drip rate and drip time to meet the application's latency and throughput needs.

Although ObliviSync works well in practice already, there are still interesting and difficult open problems in this domain. While we have optimized the efficiency for the client, cloud service providers may be hesitant to encourage systems such as ObliviSync because they will eliminate the possibility of deduplication between users, where common files are stored only once by the service provider. Furthermore, as our system only allows one ObliviSync-RW client at any given time, an important use-case of collaborative editing is not permitted here. It may be necessary to overcome challenges such as these in order to bring oblivious cloud storage into mainstream use.

\section{ACKNOWLEDGEMENTS}

This work was supported in part in part by ONR awards N0001416WX01489 and N0001416WX01645, and NSF award \#1618269, \#1406177, and \#1319994.

\section{REFERENCES}

[1] Boxcrpytor. https://www.boxcryptor.com/en.
[2] fusepy. https://github.com/terencehonles/fusepy.

[3] Panbox. http://www.sirrix.de/content/pages/Panbox.htm.

[4] Sookasa. https://www.sookasa.com/.

[5] Spideroak. https://spideroak.com/.

[6] Tresorit. https://www.tresorit.com/.

[7] Viivo. https://www.viivo.com/.

[8] Erik-Oliver Blass, Travis Mayberry, Guevara Noubir, and Kaan Onarlioglu. Toward robust hidden volumes using write-only oblivious RAM. In Gail-Joon Ahn, Moti Yung, and Ninghui Li, editors, ACM CCS 14, pages 203-214. ACM Press, November 2014.

[9] Kevin D. Bowers, Catherine Hart, Ari Juels, and Nikos Triandopoulos. PillarBox: Combating next-generation malware with fast forward-secure logging. In Proceedings of the 17th International Symposium on Research in Attacks, Intrusions and Defenses: (RAID 2014), pages 4667, Gothenburg, Sweden, 2014.

[10] A. B. Downey. The structural cause of file size distributions. In Modeling, Analysis and Simulation of Computer and Telecommunication Systems, 2001. Proceedings. Ninth International Symposium on, pages 361-370, 2001.

[11] Idilio Drago, Marco Mellia, Maurizio M. Munafo, Anna Sperotto, Ramin Sadre, and Aiko Pras. Inside Dropbox: Understanding personal cloud storage services. In Proceedings of the 2012 ACM Conference on Internet Measurement Conference, IMC '12, pages 481-494, New York, NY, USA, 2012. ACM.

[12] Dropbox, Inc. Celebrating half a billion users, 2016. https://blogs.dropbox.com/dropbox/2016/03/500-million/.

[13] EncFS. https://vgough.github.io/encfs/.

[14] ObliviSync github repository. https://github.com/oblivisync/oblivisync.

[15] Oded Goldreich and Rafail Ostrovsky. Software protection and simulation on oblivious RAMs. J. ACM, 43(3):431-473, 1996.

[16] Wassily Hoeffding. Probability inequalities for sums of bounded random variables. J. Amer. Statist. Assoc., 58:13-30, 1963.

[17] Jonathan L. Dautrich Jr., Emil Stefanov, and Elaine Shi. Burst ORAM: minimizing ORAM response times for bursty access patterns. In Proceedings of the 23rd USENIX Security Symposium, pages 749-764, 2014.

[18] Eyal Kushilevitz, Steve Lu, and Rafail Ostrovsky. On the (in)security of hash-based oblivious RAM and a new balancing scheme. In Proceedings of the twenty-third annual ACM-SIAM symposium on Discrete Algorithms, pages 143-156. SIAM, 2012.

[19] Tarik Moataz, Travis Mayberry, and Erik-Oliver Blass. Constant communication ORAM with small blocksize. In ACM CCS 15, pages 862-873. ACM Press, 2015.

[20] Thomas Ristenpart, Gabriel Maganis, Arvind Krishnamurthy, and Tadayoshi Kohno. Privacy-preserving location tracking of lost or stolen devices: Cryptographic techniques and replacing trusted third parties with DHTs. In Proceedings of the 17th USENIX Security Symposium (SECURITY 2008), pages 275-290, Berkeley, CA, USA, 2008.

[21] Daniel S. Roche, Adam J. Aviv, and Seung Geol Choi. A practical oblivious map data structure with secure deletion and history independence. In 2016 IEEE Symposium on Security and Privacy, May 2016.

[22] Elaine Shi, T.-H. Hubert Chan, Emil Stefanov, and Mingfei Li. Oblivious RAM with $o\left((\log n)^{3}\right)$ worst-case cost. In Dong Hoon Lee and Xiaoyun Wang, editors, ASIACRYPT 2011, volume 7073 of LNCS, pages 197-214. Springer, December 2011.

[23] Emil Stefanov, Marten van Dijk, Elaine Shi, Christopher W. Fletcher, Ling Ren, Xiangyao Yu, and Srinivas Devadas. Path ORAM: an extremely simple oblivious RAM protocol. In Ahmad-Reza Sadeghi, Virgil D. Gligor, and Moti Yung, editors, ACM CCS 13, pages 299-310. ACM Press, November 2013.

[24] Sandeep Tamrakar, Long Nguyen Hoang, Praveen Kumar Pendyala, Andrew Paverd, N. Asokan, and Ahmad-Reza Sadeghi. OmniShare: Securely accessing encrypted cloud storage from multiple authorized devices. CoRR, abs/1511.02119, 2015.

[25] Andrew S. Tanenbaum, Jorrit N. Herder, and Herbert Bos. File size distribution on UNIX systems: Then and now. SIGOPS Oper. Syst. Rev., 40(1):100-104, January 2006. 